\title{
Singular perturbation near mode-coupling transition
}

\author{
Mami Iwata and Shin-ichi Sasa \\ Department of Pure and Applied Sciences, University of Tokyo, Komaba, Tokyo \\ 153-8902, Japan
}

\begin{abstract}
We study the simplest mode-coupling equation which describes the time correlation function of the spherical $p$-spin glass model. We formulate a systematic perturbation theory near the mode-coupling transition point by introducing multiple time scales. In this formulation, the invariance with respect to the dilatation of time in a late stage yields an arbitrary constant in a leading order expression of the solution. The value of this constant is determined by a solvability condition associated with a linear singular equation for perturbative corrections in the late stage. The solution thus constructed provides exactly the $\alpha$-relaxation time.

PACS numbers: 05.10.-a,64.70.Q-, 02.30.Oz
\end{abstract}

\section{Introduction}

About a quarter of a century ago, a peculiar type of slow relaxation was discovered theoretically in researches of glassy systems [1, 2]. This relaxation behavior is characterized by two different time scales, both of which diverge at a temperature. An illustrative example exhibiting such a behavior is the spherical $p$-spin glass model [3]. The normalized time-correlation function $\phi(t)$ of total magnetization in this model turned out to satisfy exactly the so-called mode-coupling equation, which is written as

$$
\partial_{t} \phi(t)=-\phi(t)-g \int_{0}^{t} d s \phi^{2}(t-s) \partial_{s} \phi(s)
$$

for the case $p=3$. Here, the initial condition is given by $\phi(0)=1$, and the parameter $g$ is proportional to the square of the inverse temperature. Since this equation is derived under an assumption that the system possesses the stationarity, (1) is valid only in a regime $0 \leq g<g_{\mathrm{c}}$, where $g_{\mathrm{c}}$ will be given later.

A remarkable feature of (1) is the existence of nonlinear memory. One can regard (11) as one of the simplest equations that characterize a universality class consisting of models with nonlinear memory. Indeed, some qualitatively new features in glassy systems have been uncovered by studying (11) and its extended forms. (See Ref. [4] as a review.) In particular, two divergent time scales were found just below the transition point $g=g_{\mathrm{c}}$, and the precise values of the exponents characterizing the divergences were determined. Furthermore, extensive studies have been attempted so as to construct the solution in a systematic manner. On the basis of past achievements, in the present 
paper, we propose a perturbation method for analyzing (1), which might shed new light on the nature near the mode coupling transition.

We shall address the question we study in this paper. Let $f_{\infty}$ be the value of $\phi(t \rightarrow \infty)$. We substitute $\phi(t)=G(t)+f_{\infty}$ into (11) and take the limit $t \rightarrow \infty$. We then obtain

$$
-f_{\infty}+g f_{\infty}^{2}\left(1-f_{\infty}\right)=0
$$

where we have used the relation $G(0)=1-f_{\infty}$. From the graph $g f_{\infty}^{2}\left(1-f_{\infty}\right)$ as a function of $f_{\infty}$, we find that the non-trivial solution $\left(f_{\infty} \neq 0\right)$ appears when $g \geq g_{\mathrm{c}}=4$. This transition is called the mode-coupling transition. Note that $f_{\infty}=1 / 2$ when $g=g_{\mathrm{c}}$. Below we express this value of $f_{\infty}$ as $f_{\mathrm{c}}$. We then introduce a small positive parameter $\epsilon$ by setting $g=g_{\mathrm{c}}-\epsilon$, and we denote the solution of (1) by $\phi_{\epsilon}(t)$. In this paper, we formulate a perturbation theory for (1). As a result, we obtain an asymptotic form of $\phi_{\epsilon}(t)$ in the small $\epsilon$ limit.

More concretely, for a given small positive $\epsilon$, we want to express $\phi_{\epsilon}(t)$ in terms of $\epsilon$ and $\epsilon$-independent functions. For readers' reference, in figure 1 (left), we display the numerical solution $\phi_{\epsilon}(t)$ with $\epsilon=10^{-3}$. Here, when solving (1), we used the algorithm proposed in Ref. [5]. In figure 1 (right), we also display the $\epsilon$-dependence of the $\alpha$ relaxation time $\tau_{\alpha}$ which is defined by $\phi_{\epsilon}\left(\tau_{\alpha}\right)=1 / 4$. We want to calculate $\tau_{\alpha}$ based on our theory.
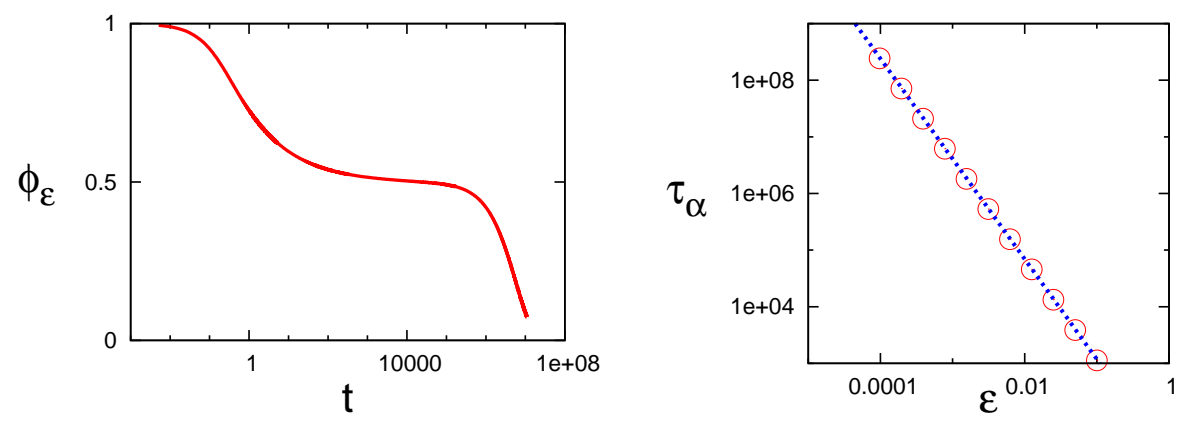

Figure 1. $\phi_{\epsilon}(t)$ with $\epsilon=10^{-3}$ (left). $\alpha$-relaxation time as a function of $\epsilon$ (right). The circle symbols represent the result of numerical simulation of (11). The dotted line corresponds to the theoretical calculation $\tau_{\alpha}=20 \epsilon^{-1.77}$ given by (43).

This paper is organized as follows. In section 2 , we set up our theory. In particular, we give a useful expression of a perturbative solution. This section also includes a review of known facts in order to have a self-contained description. In section 3 , we formulate a systematic perturbation theory on the basis of our expression, and we determine a leading order form of the solution. We check the validity of our theory by comparing our theoretical result of $\tau_{\alpha}$ with that measured by direct numerical simulations of (1). Section 4 is devoted to remarks on possible future studies. Technical details are summarized in Appendices. 


\section{Preliminaries}

\subsection{Solution with $\epsilon=0$}

We first investigate the solution $\phi_{0}(t)$. It is expressed by $\phi_{0}(t)=G_{0}(t)+f_{\mathrm{c}}$ with a function $G_{0}(t)$ which decays to 0 as $t \rightarrow \infty$. The equation for $G_{0}$ is written as

$$
\partial_{t} G_{0}(t)+f_{\mathrm{c}}+G_{0}(t)+g_{c} \int_{0}^{t} d s\left(f_{\mathrm{c}}+G_{0}(t-s)\right)^{2} \partial_{s} G_{0}(s)=0 .
$$

An asymptotic form of $G_{0}(t)$ in the large $t$ limit can be derived by employing a formula

$$
x \int_{0}^{t} d s\left[(t-s)^{-x}-t^{-x}\right] s^{-x-1}=\left(1-\frac{\Gamma^{2}(1-x)}{\Gamma(1-2 x)}\right) t^{-2 x}
$$

for any $x<1$ and $t>0$, where $\Gamma(x)$ is the Gamma function. The result is

$$
G_{0}(t) \simeq c_{0} t^{-a}
$$

where $a$ is a constant that satisfies a relation

$$
\frac{\Gamma^{2}(1-a)}{\Gamma(1-2 a)}=\frac{1}{2}
$$

The value of $a$ is estimated as $a=0.395$. In figure 2, we display the graphs of $\phi_{0}(t)$ and $G_{0}(t)$, which are calculated numerically.

As shown in Appendix A, an approximate expression of $c_{0}$ is calculated as $c_{0}^{\text {app }}=$ $(a /(1-a))^{a}(1-a) / 2^{a+1}$ by a matching procedure. Its value, 0.194 , is not far from $c_{0}=0.25$ obtained from a numerical fitting of the graph of $G_{0}(t)$. It might be possible to improve the approximation in a systematic manner. However, in this paper, we do not pursue such improvements. The important thing here is that the $\epsilon$-independent function $G_{0}(t)$ is defined with understanding of its asymptotic form.
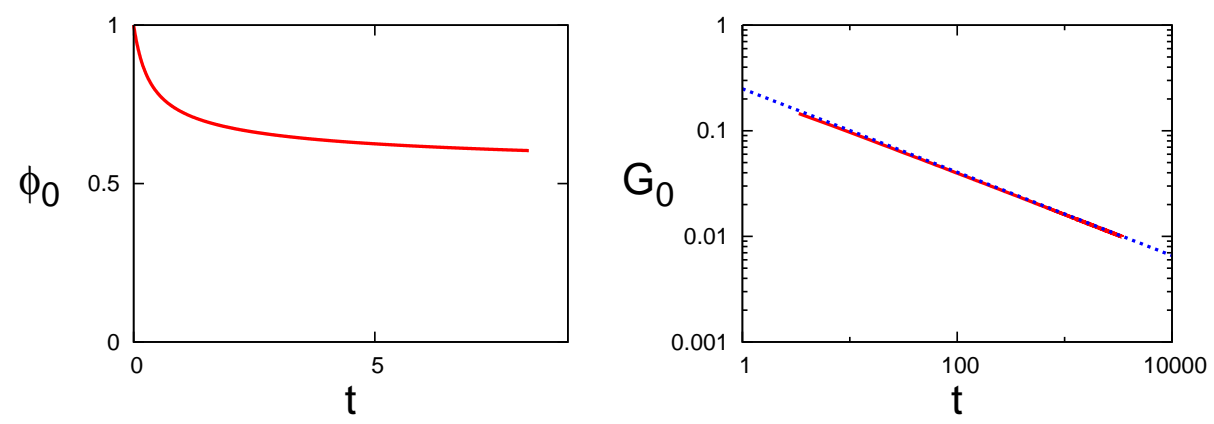

Figure 2. $\phi_{0}(t)$ (left) and $G_{0}(t)$ in the log-log plot (right). The dotted line in the right figure represents $0.25 t^{-0.395}$.

\subsection{Expression of the solution with $\epsilon>0$}

One may expect that the solution $\phi_{\epsilon}(t)$ is close to $\phi_{0}(t)$. However, recall that $\phi_{0}(t \rightarrow \infty)$ changes discontinuously from 0 to $1 / 2$ when $g$ passes at $g=g_{\text {c }}$ from below. This 
fact means that a small perturbation from $g=g_{\mathrm{c}}(\epsilon=0)$ yields a singular behavior. Examples of such singular perturbation can be seen in Refs. [6, 7].

Before formulating effects of the perturbation, we conjecture a functional form of the solution $\phi_{\epsilon}(t)$. First, $\phi_{\epsilon}(t)$ should be close to $\phi_{0}(t)$ in an early stage where $\phi_{\epsilon}>f_{\mathrm{c}}$. Since $\phi_{0}(t) \rightarrow f_{\mathrm{c}}$ as $t \rightarrow \infty$, the trajectory $\phi_{\epsilon}(t)$ stays in a region near $f_{\mathrm{c}}$ for a long time. However, since there is no non-trivial solution $f_{\infty} \neq 0$ for positive $\epsilon, \phi_{\epsilon}$ goes away from the region $\phi_{\epsilon} \simeq f_{\mathrm{c}}$ and finally approaches the origin $\phi_{\epsilon}=0$. Such a behavior is substantially different from $\phi_{0}(t)$. We thus introduce a quantity $A\left(\epsilon^{\gamma_{2}} t\right)$ that describes the relaxation behavior from $\phi_{\epsilon} \simeq f_{\mathrm{c}}$ to $\phi_{\epsilon}=0$, where $A(0)=f_{\mathrm{c}}$. The functional form of $A$ is independent of $\epsilon$, while its argument is the scaled time $t_{2}=\epsilon^{\gamma_{2}} t$ with a positive constant $\gamma_{2}$. We also expect that a switching from $\phi_{\epsilon}(t) \simeq \phi_{0}(t)$ in the early stage to $\phi_{\epsilon}(t) \simeq A\left(\epsilon^{\gamma_{2}} t\right)$ in the late stage occurs around another characteristic time of $O\left(\epsilon^{-\gamma_{1}}\right)$ with a positive constant $\gamma_{1}$. Keeping this behavior in mind, we express the solution as

$$
\phi_{\epsilon}(t)=G_{0}(t) \Theta\left(\epsilon^{\gamma_{1}} t\right)+A\left(\epsilon^{\gamma_{2}} t\right)+\varphi_{\epsilon}(t),
$$

where the switching function $\Theta$ satisfies $\Theta(0)=1$ and $\Theta(\infty)=0$. The functional form of $\Theta$ is independent of $\epsilon$, while its argument depends on $\epsilon . \varphi_{\epsilon}(t)$ represents a small correction that satisfies $\varphi_{\epsilon} \rightarrow 0$ in the limit $\epsilon \rightarrow 0$ for any $t$. $\Theta, A, \gamma_{1}, \gamma_{2}$ and $\varphi_{\epsilon}(t)$ will be determined later.

In order to have a simple description, we define a set of scaled coordinates $\left(t_{0}, t_{1}, t_{2}\right)$ on the time axis as $t_{i}=\epsilon^{\gamma_{i}} t$, where $\gamma_{0}=0$. Throughout the paper, a time coordinate with an integer subscript represents the scaled coordinate determined by the subscript. Note that $t_{0}, t_{1}$ and $t_{2}$ appear as the arguments of $G_{0}, \Theta$ and $A$, respectively. Physically, the first relaxation occurs in the early stage $t_{0} \sim O(1)$; the behavior around $\phi_{\epsilon}=f_{\mathrm{c}}$ is observed in the intermediate stage $t_{1} \sim O(1)$; and the relaxation behavior from $\phi_{\epsilon} \simeq f_{\mathrm{c}}$ to $\phi_{\epsilon}=0$ is described in the late stage $t_{2} \sim O(1)$. In researches of glassy systems, the intermediate and the late stages are termed the $\beta$-relaxation regime and the $\alpha$-relaxation regime, respectively.

\subsection{Equation for $A$}

We substitute (77) into (11) and take the limit $\epsilon \rightarrow 0$ with the scaled time $t_{2}=\epsilon^{\gamma_{2}} t$ fixed. We then obtain

$$
A\left(t_{2}\right)-g_{c}\left(1-f_{\mathrm{c}}\right) A^{2}\left(t_{2}\right)+g_{c} \int_{0}^{t_{2}} d s_{2} A^{2}\left(t_{2}-s_{2}\right) A^{\prime}\left(s_{2}\right)=0 .
$$

In this paper, the prime symbol represents the differentiation with respect to the argument of the function. The equation (88) provides the explicit definition of $A$ with the condition $A(0)=f_{\mathrm{c}}$. However, this equation cannot determine $A$ uniquely. Indeed, for a given solution $A\left(t_{2}\right)$ of (8),$A\left(\lambda t_{2}\right)$ with any positive $\lambda$ is another solution of (8)). This dilatational symmetry is a remarkable property of (8) $)$.

Here, by analyzing the short time behavior in (8), one can confirm that $A\left(t_{2}\right)-f_{\mathrm{c}}$ is proportional to $t_{2}$ in the small $t_{2}$ limit. Thus, we can choose a special solution of $A$ such that $A^{\prime}(0)=-1$. In the argument below, $A$ represents this special solution; 
and the other solutions are described by $A\left(\lambda t_{2}\right)$. For later convenience, we define $A_{\lambda}$ by $A_{\lambda}\left(t_{2}\right)=A\left(\lambda t_{2}\right)$, and $A$ in (17) is replaced with $A_{\lambda}$. In particular, we have

$$
A_{\lambda}\left(t_{2}\right)=f_{\mathrm{c}}-\lambda t_{2}+o\left(t_{2}\right)
$$

in the limit $t_{2} \rightarrow 0$. Note that $\lambda$ is an arbitrary constant until a special requirement is imposed. The functional form of $A\left(t_{2}\right)$ can be obtained by solving (8) numerically with a simple discretization of time. We display the graph of $A\left(t_{2}\right)$ in figure 3 , It should be noted here that the mathematical determination of the functional form is not the heart of the problem. The important thing is that the $\epsilon$-independent function $A$ is defined without any ambiguities.
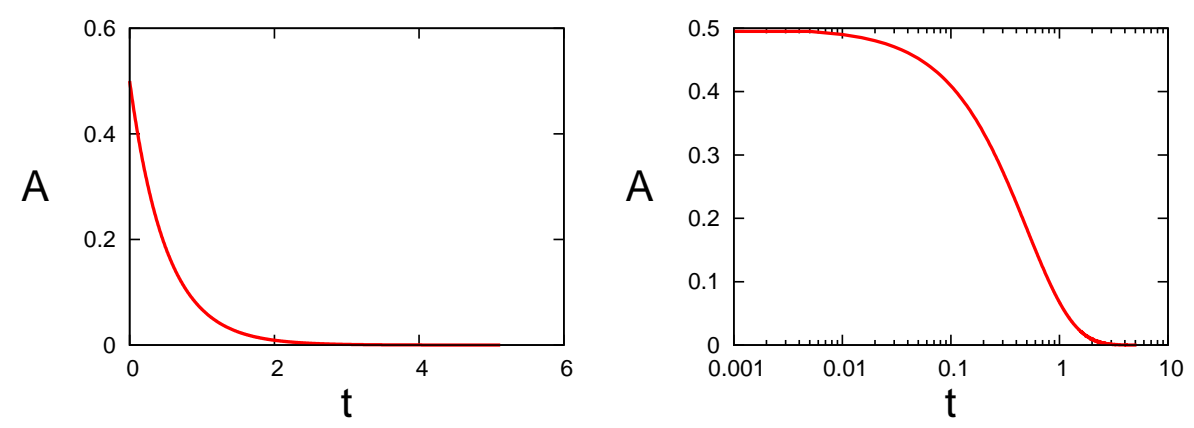

Figure 3. $A(t)$ (left) and its semi-log plot (right).

We express the dilatational symmetry in terms of a mathematical equality. Let us define

$$
\Phi_{2}\left(t_{2} ; A_{\lambda}\right) \equiv A_{\lambda}\left(t_{2}\right)-g_{\mathrm{c}}\left(1-f_{\mathrm{c}}\right) A_{\lambda}^{2}\left(t_{2}\right)+g_{\mathrm{c}} \int_{0}^{t_{2}} d s_{2} A_{\lambda}^{2}\left(t_{2}-s_{2}\right) A_{\lambda}^{\prime}\left(s_{2}\right) \cdot(10
$$

Since (8) is identical to $\Phi_{2}\left(t_{2} ; A\right)=0$, the dilatational symmetry is expressed by $\Phi\left(t_{2} ; A_{\lambda}\right)=0$ for any $\lambda$. Then, taking the derivative with respect to $\lambda$, we obtain

$$
\int_{0}^{\infty} d s_{2} L_{A}\left(t_{2}, s_{2}\right) \partial_{\lambda} A_{\lambda}\left(s_{2}\right)=0
$$

where $L_{A}$ is the linearized operator around $A_{\lambda}$, which is defined by

$$
L_{A}\left(t_{2}, s_{2}\right)=\frac{\delta \Phi_{2}\left(t_{2} ; A_{\lambda}\right)}{\delta A_{\lambda}\left(s_{2}\right)} .
$$

Its explicit form is given by

$$
\begin{aligned}
L_{A}\left(t_{2}, s_{2}\right)= & \delta\left(t_{2}-s_{2}\right)\left(1-2 g_{\mathrm{c}}\left(1-f_{\mathrm{c}}\right) A_{\lambda}\left(s_{2}\right)+g_{\mathrm{c}} f_{\mathrm{c}}^{2}\right) \\
& +g_{\mathrm{c}} \theta\left(t_{2}-s_{2}\right) 2 A_{\lambda}^{\prime}\left(t_{2}-s_{2}\right)\left(A_{\lambda}\left(s_{2}\right)+A_{\lambda}\left(t_{2}-s_{2}\right)\right) .
\end{aligned}
$$

It will be found below that (11) plays a key role in our formulation.

Now, from the definition of $\gamma_{1}$, we have $f_{\mathrm{c}}+G_{0}\left(\epsilon^{-\gamma_{1}}\right) \simeq A_{\lambda}\left(\epsilon^{\gamma_{2}-\gamma_{1}}\right)$. By substituting (5) and (9) into this relation, we obtain

$$
\gamma_{1}=\frac{\gamma_{2}}{1+a}
$$




\subsection{Functional form of $\Theta$}

We can formulate a systematic perturbation theory with employing an arbitrary switching function $\Theta\left(t_{1}\right)$ when it decays faster than a power-law function $t_{1}^{-1+a}$, as we will see in the next section. For example, one can choose a physically reasonable form

$$
\Theta\left(t_{1}\right)=\exp \left(-t_{1} / t_{\mathrm{c}}\right),
$$

where $t=t_{\mathrm{c}} \epsilon^{-\gamma_{1}}$ corresponds to the time when the graph $\phi_{0}(t)$ is closest to that of $A_{\lambda}\left(\epsilon^{\gamma_{2}} t\right)$. That is, $t_{\mathrm{c}}$ satisfies $a c_{0} t_{\mathrm{c}}^{-a-1}=\lambda$. Note, however, that there is no reason that we must choose this form. Indeed, other forms such as $\Theta\left(t_{1}\right)=\exp \left(-2 t_{1} / t_{\mathrm{c}}\right)$ and $\Theta\left(t_{1}\right)=\exp \left(-\left(t_{1} / t_{\mathrm{c}}\right)^{2}\right)$ might also be physically reasonable to the same extent as (15)). Of course, the final result should be independent of the choice of the functional form.

\subsection{Summary}

In our formulation, we set the unperturbative solution $\phi_{\mathrm{u}}$ as

$$
\phi_{\mathrm{u}}(t)=G_{0}(t) \Theta\left(\epsilon^{\gamma_{1}} t\right)+A_{\lambda}\left(\epsilon^{\gamma_{2}} t\right),
$$

and we express the perturbative solution $\phi_{\epsilon}$ by

$$
\phi_{\epsilon}(t)=\phi_{\mathrm{u}}(t)+\varphi_{\epsilon}(t) .
$$

$G_{0}$ and $A$ are already determined. $\gamma_{1}$ is connected with $\gamma_{2}$ in (14). $\Theta$ is assumed to take an arbitrary form. Thus, the problem we solve is the determination of $\gamma_{2}$ and $\lambda$ as well as the perturbative calculation of the correction $\varphi_{\epsilon}(t)$. Note that $\gamma_{2}$ and $\lambda$ appear in the leading order expression of the solution. In particular, since the value of $\lambda$ has never been known, the calculation of $\lambda$ is a cornerstone of our theory.

\section{Systematic perturbation}

\section{1. preliminary}

For any trajectory $\psi(t)$ with $\psi(0)=1$, we define

$$
F_{\epsilon}(t ; \psi) \equiv \partial_{t} \psi+\psi+g \int_{0}^{t} d s \psi^{2}(t-s) \partial_{s} \psi(s) .
$$

Let $L_{\epsilon}(t, s ; \psi)$ be the linearized operator of $F_{\epsilon}(t ; \psi)$, which is defined by

$$
L_{\epsilon}(t, s ; \psi)=\frac{\delta F_{\epsilon}(t ; \psi)}{\delta \psi(s)} .
$$

Its explicit form is written as

$$
\begin{aligned}
L_{\epsilon}(t, s ; \psi)= & \delta(t-s)(1+g)+\delta^{\prime}(t-s) \\
& -g \theta(t-s)\left(\partial_{s} \psi^{2}(t-s)-2 \psi(s) \psi^{\prime}(t-s)\right) .
\end{aligned}
$$

The mode-coupling equation (11) is expressed by

$$
F_{\epsilon}\left(t ; \phi_{\epsilon}\right)=0 \text {. }
$$




\subsection{Calculation}

The substitution of (17) into (21) yields non-trivial $\epsilon$ dependences through the evaluation of the integration term in (18) with using the scaled coordinates. In order to avoid a complicated description, we focus our presentation on an important part of the calculation.

We first evaluate $F_{\epsilon}\left(\epsilon^{-\gamma_{2}} t_{2} ; \phi_{\mathrm{u}}\right)$ in the small $\epsilon$ limit with $t_{2}$ fixed. As explained in Appendix B, we derive

$$
F_{\epsilon}\left(\epsilon^{-\gamma_{2}} t_{2} ; \phi_{\mathrm{u}}\right) \simeq \epsilon \frac{1}{g_{\mathrm{c}}} A_{\lambda}\left(t_{2}\right)+\epsilon^{\gamma_{2}-\gamma_{1}(1-a)} 2 g_{\mathrm{c}}\left(f_{\mathrm{c}}+A_{\lambda}\left(t_{2}\right)\right) A_{\lambda}^{\prime}\left(t_{2}\right) c_{0} \theta,
$$

where higher order terms of $O\left(\epsilon^{\gamma_{2}-\gamma_{1}(1-2 a)}\right)$ are neglected, and $\theta$ is a constant determined by

$$
\theta=\int_{0}^{\infty} d s s^{-a} \Theta(s) .
$$

We here make three important remarks on (22). First, if we did not introduce the switching function $\Theta$ in the expression of the solution (7), we would have a form rather different from (22), for which the analysis seems to be hard. Second, the function $\Theta$ should provide a finite value of $\theta$. This means that $\Theta\left(t_{1}\right)$ should decay faster than a power-law function $t_{1}^{-1+a}$. Third, the two terms in (22) should balance each other. Otherwise, a contradiction occurs. (See an argument below (37).) The last remark leads to the relation $\gamma_{2}-\gamma_{1}(1-a)=1$. By combining it with (14), we obtain well-known results

$$
\gamma_{1}=\frac{1}{2 a}
$$

and

$$
\gamma_{2}=\frac{1}{2 a}+\frac{1}{2}
$$

which correspond to the exponents characterizing divergences of the $\beta$-relaxation time and the $\alpha$-relaxation time in glassy systems, respectively. Then, (22) with (24) and (25) becomes

$$
F_{\epsilon}\left(\epsilon^{-\gamma_{2}} t_{2} ; \phi_{\mathrm{u}}\right)=\epsilon \mathcal{F}_{2}^{(1)}\left(t_{2}\right)+O\left(\epsilon^{3 / 2}\right),
$$

where $\mathcal{F}_{2}^{(1)}$ is the $\epsilon$-independent function given by

$$
\mathcal{F}_{2}^{(1)}\left(t_{2}\right)=\frac{1}{g_{\mathrm{c}}} A_{\lambda}\left(t_{2}\right)+2 g_{\mathrm{c}}\left(f_{\mathrm{c}}+A_{\lambda}\left(t_{2}\right)\right) A_{\lambda}^{\prime}\left(t_{2}\right) c_{0} \theta
$$

Furthermore, we can prove

$$
\epsilon^{-\gamma_{2}} L_{\epsilon}\left(\epsilon^{-\gamma_{2}} t_{2}, \epsilon^{-\gamma_{2}} s_{2} ; \phi_{\mathrm{u}}\right)=L_{A}\left(t_{2}, s_{2}\right)+O(\epsilon),
$$

where $L_{A}$ is given by (13).

Now, let us compare (26) and (21) with (17). We then find that the perturbative correction is expressed as

$$
\varphi_{\epsilon}\left(\epsilon^{-\gamma_{2}} t_{2}\right)=\epsilon \bar{\varphi}_{2}^{(1)}\left(t_{2}\right)+O\left(\epsilon^{3 / 2}\right)
$$


in the regime $t_{2} \sim O(1)$ with the limit $\epsilon \rightarrow 0$. We also write

$$
\varphi_{\epsilon}\left(\epsilon^{-\gamma_{1}} t_{1}\right)=\epsilon^{\alpha} \bar{\varphi}_{1}^{(\alpha)}\left(t_{1}\right)+o\left(\epsilon^{\alpha}\right)
$$

in the regime $t_{1} \sim O(1)$ with the limit $\epsilon \rightarrow 0$, where $\alpha$ is a positive constant. Then, (21) with (17) becomes

$$
F_{\epsilon}\left(\epsilon^{-\gamma_{2}} t_{2} ; \phi_{\mathbf{u}}+\epsilon^{\alpha} \bar{\varphi}_{1}^{(\alpha)}\right)+\epsilon \int_{0}^{\infty} d s_{2} L_{A}\left(t_{2}, s_{2}\right) \bar{\varphi}_{2}^{(1)}\left(s_{2}\right)=o(\epsilon),
$$

where the contribution of $\varphi_{\epsilon}\left(t_{0}\right)$ is included in the right-hand side. Here, by an argument similar to Appendix B, we can estimate

$$
F_{\epsilon}\left(\epsilon^{-\gamma_{2}} t_{2} ; \phi_{\mathrm{u}}+\epsilon^{\alpha} \bar{\varphi}_{1}^{(\alpha)}\right)=\epsilon \mathcal{F}_{2}^{(1)}\left(t_{2}\right)+O\left(\epsilon^{\alpha+1 / 2}\right) .
$$

In order to describe a theoretical framework in a simple manner, for the moment, we focus on the case $\alpha>1 / 2$. The other case $\alpha \leq 1 / 2$ will be discussed in section 3.4. The equation for $\bar{\varphi}_{2}^{(1)}\left(t_{2}\right)$ is then simply written as

$$
\int_{0}^{\infty} d s_{2} L_{A}\left(t_{2}, s_{2}\right) \bar{\varphi}_{2}^{(1)}\left(s_{2}\right)=-\mathcal{F}_{2}^{(1)}\left(t_{2}\right) .
$$

\subsection{Solvability condition}

We notice that (33) is a linear equation for $\bar{\varphi}_{2}^{(1)}$, which is singular because there exists the zero eigenvector $\Phi_{0}=\partial_{\lambda} A_{\lambda}$ associated with the dilatational symmetry (11). Let $\Phi_{0}^{\dagger}$ be the adjoint zero eigenvector that satisfies

$$
\int_{0}^{\infty} d s_{2} L_{A}\left(s_{2}, t_{2}\right) \Phi_{0}^{\dagger}\left(s_{2}\right)=0
$$

Then, there exists a solution of (33) only when the condition

$$
\int_{0}^{\infty} d t_{2} \Phi_{0}^{\dagger}\left(t_{2}\right) \mathcal{F}_{2}^{(1)}\left(t_{2}\right)=0
$$

is satisfied. Otherwise, (33) leads to $0 \neq 0$ and hence there is no solution $\bar{\varphi}_{2}^{(1)}$. The equality (35) is called the solvability condition for the singular equation (33). Note, however, that the solvability condition is not satisfied as an identity. Here, let us recall that $\lambda$ is still an arbitrary constant. Thus, we are allowed to determine the value of $\lambda$ so that the solvability condition (35) can be satisfied. Only for this special value of $\lambda$, the perturbation theory can be formulated consistently.

Concretely, since we find that $\Phi_{0}^{\dagger}\left(s_{2}\right)=\delta\left(s_{2}\right)$ from (34) with (13)), the solvability condition (35) becomes

$$
\mathcal{F}_{2}^{(1)}(0)=0 .
$$

The explicit form $\mathcal{F}_{2}^{(1)}(0)$ obtained from (27) leads to

$$
\lambda=\frac{1}{64 c_{0} \theta} .
$$

Here, let us go back to (22). If $\gamma_{2}-\gamma_{1}(1-a)$ were not equal to 1 , the condition (361) could not be satisfied for any positive $\lambda$. In this sense, one can regard that the solvability condition determines the exponent $\gamma_{2}$ as well as the constant $\lambda$. 


\subsection{Determination of $\lambda$}

Apparently, (37) shows that $\lambda$ depends on the choice of $\Theta$. However, the situation is a little bit complicated. We shall explain the way how to determine the value of $\lambda$ in detail.

We study the case $\epsilon \rightarrow 0$ with $t_{1}$ fixed. In this limit, (7) can be expressed as

$$
\phi_{\epsilon}\left(\epsilon^{-\gamma_{1}} t_{1}\right)=f_{\mathrm{c}}+\epsilon^{1 / 2}\left[c_{0} t_{1}^{-a} \Theta\left(t_{1}\right)-\lambda t_{1}\right]+\varphi_{\epsilon}\left(\epsilon^{-\gamma_{1}} t_{1}\right)
$$

where we have used (5) and (9). Since $\Theta$ is arbitrary, $\varphi_{\epsilon}\left(\epsilon^{-\gamma_{1}} t_{1}\right)$ includes a term of $O\left(\epsilon^{1 / 2}\right)$ unless a special $\Theta$ is employed. This means that $\alpha$ in (30) is equal to $1 / 2$. As is seen from (32), when $\alpha=1 / 2, \bar{\varphi}_{2}^{(1)}\left(t_{2}\right)$ must be calculated with taking account of $\bar{\varphi}_{1}^{(1 / 2)}$. Concretely, the right hand side of (3ㅏ) should be replaced with $-\tilde{\mathcal{F}}_{2}^{(1)}\left(t_{2}\right)$, where

$$
F_{\epsilon}\left(\epsilon^{-\gamma_{2}} t_{2} ; \phi_{\mathrm{u}}+\epsilon^{1 / 2} \bar{\varphi}_{1}^{(1 / 2)}\right)=\epsilon \tilde{\mathcal{F}}_{2}^{(1)}\left(t_{2}\right)+o(\epsilon) .
$$

Then, the solvability condition (36) is also replaced with $\tilde{\mathcal{F}}_{2}^{(1)}(0)=0$. Without the replacement, (37) provides nothing more than an approximation of $\lambda$. For example, (37) with (15) leads to $\lambda=\left[(64 \Gamma(1-a))^{(1+a) /(2 a)} c_{0}^{1 / a} a^{(1-a) /(2 a)}\right]^{-1} \simeq 0.022$ as one approximate value.

Now, let us calculate the precise value of $\lambda$. One natural method is to choose a functional form of $\Theta$ so that the condition $\alpha>1 / 2$ is satisfied. We denote this special $\Theta$ by $\Theta_{*}$. Then, for a given $\Theta$, the correction $\bar{\varphi}_{1}^{(1 / 2)}$ is determined by

$$
c_{0} t_{1}^{-a} \Theta\left(t_{1}\right)+\bar{\varphi}_{1}^{(1 / 2)}\left(t_{1}\right)=c_{0} t_{1}^{-a} \Theta_{*}\left(t_{1}\right) .
$$

Therefore, (36) using $\Theta_{*}$ is equivalent to $\tilde{\mathcal{F}}_{2}^{(1)}(0)=0$ using $\Theta$. In other words, the precise calculation of $\lambda$ starting from $\Theta$ can be done through $\Theta_{*}$. This also indicates explicitly that the final and precise result is independent of the choice of $\Theta$. In any cases, the problem is focused on the calculation of $\Theta_{*}$.

As explained in Appendix C, we can derive the equation for $Q\left(t_{1}\right) \equiv c_{0} t_{1}^{-a} \Theta_{*}\left(t_{1}\right)$ in the form

$$
\begin{aligned}
& \frac{1}{8}-8 \lambda \int_{0}^{t_{1}} d s_{1}\left[Q\left(s_{1}\right)-Q\left(t_{1}\right) / 2\right] \\
& +2 Q^{2}+4 \int_{0}^{t_{1}} d s_{1}\left[Q\left(t_{1}-s_{1}\right)-Q\left(t_{1}\right)\right] Q^{\prime}\left(s_{1}\right)=0 .
\end{aligned}
$$

We study this equation by regarding $\lambda$ as a parameter whose value is not specified beforehand. We denote this solution by $Q\left(t_{1} ; \lambda\right)$. For almost all $\lambda, Q\left(t_{1} ; \lambda\right)$ is not bounded as $t_{1} \rightarrow \infty$, while there exists the special value $\lambda_{*}$ such that $Q\left(t_{1} ; \lambda_{*}\right) \rightarrow 0$ as $t_{1} \rightarrow \infty$. A necessary condition for this property is easily derived by considering the limit $t_{1} \rightarrow \infty$ in (41):

$$
\lambda_{*}=\frac{1}{64}\left[\int_{0}^{\infty} d s_{1} Q\left(s_{1} ; \lambda_{*}\right)\right]^{-1} .
$$

This is equivalent to the expression (37) that determines the value of $\lambda$ by the solvability condition (36) under the assumption $\alpha>1 / 2$. Therefore, once we find $\lambda_{*}$ such that 
$Q\left(t_{1} ; \lambda_{*}\right) \rightarrow 0$ as $t_{1} \rightarrow \infty$, this $\lambda_{*}$ is the precise value of $\lambda$ that we want to have. Simultaneously, we obtain $\Theta_{*}\left(t_{1}\right)$ from $Q\left(t_{1} ; \lambda_{*}\right)$.

The problem of finding $\lambda_{*}$ is investigated by a shooting method. We first solve (41) numerically for a given $\lambda$. Basically, we employ a simple discretization method. In order to treat properly the singular behavior near $t=0$, we utilize the result of the short time expansion of $Q\left(t_{1} ; \lambda\right)$ near $t=0$. (See Appendix D for the short time expansion.) Suppose that we already investigated the system with $\lambda_{k}, k=0,1, \cdots, n$. We here note that $Q\left(t_{1} ; \lambda\right) \rightarrow-\infty$ as $t_{1} \rightarrow \infty$ when $\lambda=0$ and that $Q\left(t_{1} ; \lambda\right) \rightarrow \infty$ as $t_{1} \rightarrow \infty$ when $\lambda$ is sufficiently large. Based on this observation, we define $\underline{\mu}_{n} \equiv \max \lambda_{k}$ such that $Q\left(t_{1} ; \lambda_{k}\right) \rightarrow-\infty$ as $t_{1} \rightarrow \infty$, and $\bar{\mu}_{n} \equiv \min \lambda_{k}$ such that $Q\left(t_{1} ; \lambda_{k}\right) \rightarrow \infty$ as $t_{1} \rightarrow \infty$. We then choose $\lambda_{n+1}$ as $\lambda_{n+1}=\left(\underline{\mu}_{n}+\bar{\mu}_{n}\right) / 2$. Starting from $\lambda_{0}=0$ and $\lambda_{1}=1$, we can determine the sequence $\left\{\lambda_{n}\right\}$ for which $\lambda_{\infty}=\lim _{n \rightarrow \infty} \lambda_{n}$ exists. From the construction method, $Q\left(t_{1} ; \lambda_{\infty}\right) \rightarrow 0$ as $t_{1} \rightarrow \infty$. Therefore, $\lambda_{*}$ is given by $\lambda_{\infty}$. By performing this procedure numerically, we estimate $\lambda_{*}=0.017$. In this manner, we have determined the precise value of $\lambda$ and the function $\Theta_{*}$. We display the functional form of $\Theta_{*}$ in figure 4.
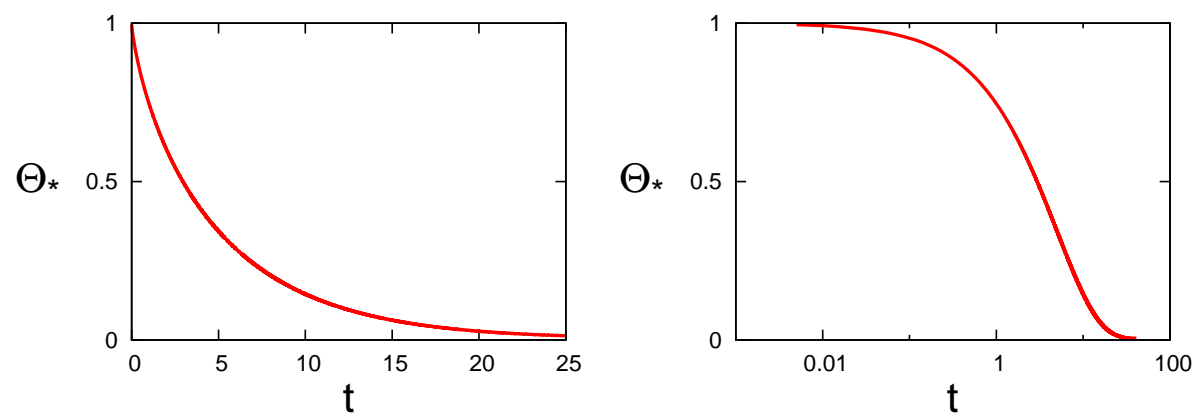

Figure 4. $\Theta_{*}(t)$ (left) and its semi-log plot (right).

\subsection{Remarks}

At the end of this section, we make two remarks. First, as a demonstration of our result, we study the $\alpha$-relaxation time $\tau_{\alpha}$ defined by $\phi_{\epsilon}\left(\tau_{\alpha}\right)=1 / 4$. Let $\tau_{A}$ be $A\left(\tau_{A}\right)=1 / 4$. Then, from the expression of the solution (17), $\tau_{\alpha}$ is estimated as $\tau_{\alpha}=\left(\tau_{A} / \lambda\right) \epsilon^{-\gamma_{2}}$. By using the value $\tau_{A}=0.346$ obtained from numerical integration of (8), we arrive at the theoretical prediction

$$
\tau_{\alpha}=20 \epsilon^{-1.77} .
$$

In figure1 (right), we display the result of numerical simulations of (1) with $\epsilon=0.1 \times 2^{-j}$, $j=0, \cdots, 10$. The numerical data are perfectly placed on the theoretical result (43)). This is an evidence that the expression (37) is correct.

The second remark is on the systematic formulation. In principle, higher order terms such as $\bar{\varphi}_{j}^{(3 / 2)}\left(t_{j}\right)$ and $\bar{\varphi}_{j}^{\left(\gamma_{2}\right)}\left(t_{j}\right)$ can also be calculated in a manner similar to that 
described in sections 3.2 and 3.3 . Such a perturbation theory with using a solvability condition has been employed in many problems [8, 9, 10,

\section{Concluding remarks}

We have formulated a systematic perturbation theory for (1). Due to the dilatational symmetry (11), an arbitrary constant $\lambda$ appears in the unperturbed solution $\phi_{\mathrm{u}}(t)$. Then, the value of $\lambda$ is determined by the solvability condition (35) associated with the linear equation (33) for the perturbative correction $\bar{\varphi}_{2}^{(1)}$. The advantage of our systematic perturbation is in a possibility of developing new and important directions. Concretely, following the three problems will be studied soon.

The first problem is to derive the fluctuation intensity of $\hat{C}\left(t, t^{\prime}\right)=$ $\sum_{j k} \sigma_{j}(t) \sigma_{k}\left(t^{\prime}\right) / N$ just below the mode-coupling transition point for the spherical $p$-spin glass, where $\sigma_{j}$ is a real spin variable that satisfies the spherical constraint $\sum_{j=1}^{N} \sigma_{j}^{2}=1$. Note that $\phi\left(t-t^{\prime}\right)=C\left(t-t^{\prime}\right) / C(0)$ with $C\left(t-t^{\prime}\right)=\left\langle\hat{C}\left(t, t^{\prime}\right)\right\rangle$ in the stationary regime. In a straightforward approach, one may study an effective potential for $\hat{C}\left(t, t^{\prime}\right)$ [11]. Indeed, by employing a diagrammatic expansion with neglecting vertex corrections, the singular behavior of the effective potential was evaluated [12. Then, since the minimum of the potential corresponds to the solution of (1), the existence of the dilatational symmetry yields the Goldstone mode which carries a divergent part of fluctuations in the late stage. More explicitly, $\lambda$ in our expression is treated as a fluctuating quantity, and it is identified with the Goldstone mode. (A Related description of fluctuations near another type of bifurcation points can be seen in Refs. [13, 14.) The analysis along this line will shed a new light to the understanding of fluctuations near mode-coupling transition points.

As an alternative approach to the description of fluctuations near the mode-coupling transition, response properties against an auxiliary external field conjugated to $\hat{C}$ [15] and against a one-body potential field [16] were investigated. Their studies successfully derived the scaling form of a singular part of the fluctuation intensity of $\hat{C}$ based on an idea that such response functions are related to the fluctuation intensity. The extension of our work so as to describe the responses may provide a more quantitative result than the scaling form. Such an extension is related to a study of dynamical behavior in the aging regime, because its behavior is described by a coupled equation of the time correlation function $C\left(t, t^{\prime}\right)$ and the response function $R\left(t, t^{\prime}\right)$, which are functions of two times [17. In addition to a complicated structure of the equation, the dilatational symmetry is replaced with the time reparameterization symmetry. Since the symmetry is much wider than the dilatational symmetry, several new features may appear in the analysis. See Ref. [18] as a review for the argument on the basis of the time reparameterization symmetry.

The third problem is to analyze a rather wide class of systems with nonlinear memory. The qualitative change of the solution $f_{\infty}$ of (21) is the same type as that 
observed in an elementary saddle-node bifurcation [19]. Despite this similarity, the dynamical behavior near the saddle-node bifurcation is much simpler than that of (1) owing to the lack of nonlinear memory. Note that an edge deletion process of $k$-core percolation in a random graph is precisely described by a saddle-node bifurcation [20] and it has been pointed out that $k$-core percolation problems are related to jamming transitions [21]. Since nonlinear memory effects might appear in jamming transitions, it is important to study a mixed type of dynamical systems which connect the elementary saddle-node bifurcation with the mode-coupling transition. The calculation presented in this paper may be useful in the analysis of such models.

By studying these problems, we will have deeper understanding of slow relaxation with nonlinear memory. We also hope that this paper provokes mathematical studies of the simplest mode-coupling equation (1).

\section{Acknowledgments}

This work was supported by a grant from the Ministry of Education, Science, Sports and Culture of Japan, No. 19540394. Mami Iwata acknowledges the support by Hayashi memorial foundation for female natural scientists.

\section{Appendix A. Approximate expression of $c_{0}$}

We perform a short time expansion

$$
G_{0}(t)=\sum_{n=0}^{\infty} g_{n} t^{n},
$$

which is valid around $t=0$. All the coefficients $g_{n}$ can be determined from a recursive formula. Concretely, $g_{0}=1 / 2, g_{1}=-1$ and $g_{2}=5 / 2$. Respecting the lowest order result $G_{0}(t)=g_{0}+g_{1} t$, we assume

$$
G_{0}(t)=\frac{1}{2(1+2 t)}
$$

for $t \leq t_{*}$, where $t_{*}$ will be determined later. On the other hand, from the asymptotic form

$$
G_{0}(t)=c_{0} t^{-a}
$$

in the limit $t \rightarrow \infty$, we assume $G_{0}(t)=c_{0} t_{*}^{-a}$ for $t \geq t_{*}$. Since $G_{0}(t)$ is smooth at $t=t_{*}$, we have

$$
\begin{aligned}
& \frac{1}{1+2 t_{*}}=2 c_{0} t_{*}^{-a}, \\
& \frac{2}{\left(1+2 t_{*}\right)^{2}}=2 a c_{0} t_{*}^{-a-1} .
\end{aligned}
$$

These equations lead to $t_{*}=a /[(1-a) 2]$ and $c_{0}=(a /(1-a))^{a}(1-a) / 2^{a+1}$. 
Singular perturbation near mode-coupling transition

\section{Appendix B. Derivation of $\mathcal{F}_{2}^{(1)}$}

We shall extract a leading order contribution of $F_{2}\left(\epsilon^{-\gamma_{2}} t_{2} ; \phi_{\mathrm{u}}\right)$ in the limit $\epsilon \rightarrow 0$. In order to simplify the calculation, we utilize an identity

$$
\begin{aligned}
\int_{0}^{t} d s f(t-s) g^{\prime}(s)= & \int_{t / 2}^{t} d s\left[f(t-s) g^{\prime}(s)+g(t-s) f^{\prime}(s)\right] \\
& -f(t) g(0)+f(t / 2) g(t / 2)
\end{aligned}
$$

which plays a key role in an efficient numerical integration algorithm for solving modecoupling equations [5]. By substituting (16) into (18) and using this identity, we obtain

$$
\begin{aligned}
& F_{\epsilon}\left(\epsilon^{-\gamma_{2}} t_{2} ; \phi_{\mathrm{u}}\right)=A_{\lambda}^{\prime}\left(t_{2}\right)+A_{\lambda}\left(t_{2}\right)-g\left[A_{\lambda}^{2}\left(t_{2}\right)-A_{\lambda}^{3}\left(t_{2} / 2\right)\right] \\
& +g \int_{t_{2} / 2}^{t_{2}} d s_{2} A_{\lambda}^{\prime}\left(s_{2}\right)\left[\phi_{\mathrm{u}}^{2}\left(\epsilon^{-\gamma_{2}}\left(t_{2}-s_{2}\right)\right)+2 A_{\lambda}\left(s_{2}\right) \phi_{\mathrm{u}}\left(\epsilon^{-\gamma_{2}}\left(t_{2}-s_{2}\right)\right)\right] .
\end{aligned}
$$

Here, we take $\Delta t$ satisfying $\epsilon^{\gamma_{2}-\gamma_{1}} \ll \Delta t \ll 1$ such that $\phi_{\mathrm{u}}\left(\epsilon^{-\gamma_{2}} t_{2}\right) \simeq A_{\lambda}\left(t_{2}\right)$ in the regime $\Delta t \leq t_{2} \leq \infty$. More explicitly, we assume $\Delta t=\epsilon^{\gamma^{\prime}}$ with $\gamma_{2}-\gamma_{1}>\gamma^{\prime}>0$. We divide the integration regime in the second line of (B.2) into two parts, $\left[t_{2} / 2, t_{2}-\Delta t\right]$ and $\left[t_{2}-\Delta t, t_{2}\right]$. Let $I_{1}$ and $I_{2}$ be the integration values over the former and the latter region, respectively. By a straightforward calculation, we can estimate $I_{2}$ as

$$
\begin{aligned}
I_{2} \simeq & 2 g A_{\lambda}^{\prime}\left(t_{2}\right)\left(f_{\mathrm{c}}+A_{\lambda}\left(t_{2}\right)\right) \epsilon^{\gamma_{2}} \int_{0}^{\epsilon^{-\gamma_{2}} \Delta t} d s G_{0}(s) \Theta\left(\epsilon^{\gamma_{1}} s\right) \\
& +g \int_{t_{2}-\Delta t}^{t_{2}} d s_{2} A_{\lambda}^{\prime}\left(s_{2}\right)\left(A_{\lambda}^{2}\left(t_{2}-s_{2}\right)+2 A_{\lambda}\left(t_{2}-s_{2}\right) A_{\lambda}\left(s_{2}\right)\right)
\end{aligned}
$$

in the lowest order evaluation. We next combine the second line of $(\underline{B .3})$ with $I_{1}$ and return it to the original form. As the result, we obtain

$$
\begin{aligned}
F_{\epsilon}\left(\epsilon^{-\gamma_{2}} t_{2} ; \phi_{\mathrm{u}}\right) \simeq & A_{\lambda}\left(t_{2}\right)-g\left(1-f_{\mathrm{c}}\right) A_{\lambda}^{2}\left(t_{2}\right)+g \int_{0}^{t_{2}} d s_{2} A_{\lambda}^{2}\left(t_{2}-s_{2}\right) A_{\lambda}{ }^{\prime}\left(s_{2}\right) \\
& +2 g A_{\lambda}^{\prime}\left(t_{2}\right)\left(f_{\mathrm{c}}+A_{\lambda}\left(t_{2}\right)\right) \epsilon^{\gamma_{2}} \int_{0}^{\epsilon^{-\gamma_{2}} \Delta t} d s G_{0}(s) \Theta\left(\epsilon^{\gamma_{1}} s\right),(\mathrm{B} .4)
\end{aligned}
$$

where higher order terms are ignored. With the aid of (8), we rewrite the first line of (B.4) as $\epsilon A\left(\lambda t_{2}\right) / g_{\mathrm{c}}$. Furthermore, from an estimation

$$
\int_{0}^{\epsilon^{-\gamma_{2} \Delta t}} d s G_{0}(s) \Theta\left(\epsilon^{\gamma_{1}} s\right) \simeq c_{0} \epsilon^{-\gamma_{1}(1-a)} \int_{0}^{\infty} d s s^{-a} \Theta(s),
$$

which is valid in the limit $\epsilon \rightarrow 0$, the second line of $(\underline{B .4})$ turns out to be of $O\left(\epsilon^{\gamma_{2}-\gamma_{1}(1-a)}\right)$. These results lead to (27). We also find that the higher order terms we have neglected in (B.4) are of $O\left(\epsilon^{\gamma_{2}-\gamma_{1}(1-2 a)}\right)$ by an estimation similar to (B.5).

\section{Appendix C. Derivation of (41)}

We take $\Delta t=\epsilon^{-\alpha^{\prime}}$, where $\alpha^{\prime}$ satisfies $\alpha^{\prime}<\gamma_{1}-1 / 2$. We also define

$$
w\left(t_{1}\right) \equiv c_{0} t_{1}^{-a} \Theta_{*}\left(t_{1}\right)-\lambda t_{1} .
$$


Then, for sufficiently small $\epsilon, h_{\epsilon}(t) \equiv \phi_{\epsilon}(t)-f_{\text {c }}$ is expressed by

$$
h_{\epsilon}(t)=G_{0}(t)+O(\epsilon)
$$

for $0 \leq t \leq \Delta t$, and

$$
h_{\epsilon}(t)=\epsilon^{1 / 2} w\left(\epsilon^{\gamma_{1}} t\right)+\epsilon^{\alpha} \bar{\varphi}_{1}^{(\alpha)}\left(\epsilon^{\gamma_{1}} t\right)
$$

for $\Delta t \leq t \ll \epsilon^{-\gamma_{2}}$.

By substituting $\phi_{\epsilon}(t)=f_{\mathrm{c}}+h_{\epsilon}(t)$ into (11), we can write the equation for $h_{\epsilon}(t)$. The further substitution of (C.2) and (C.3) into the obtained equation for $h_{\epsilon}$ yields

$$
\begin{aligned}
& \epsilon\left(2 w^{2}(t)+1 / 8+4 \int_{\Delta t \epsilon^{\gamma_{1}}}^{t_{1}-\Delta t \epsilon^{\gamma_{1}}} d s_{1}\left(w\left(t_{1}-s_{1}\right)-w\left(t_{1}\right)\right) w^{\prime}\left(s_{1}\right)\right) \\
& =O\left(\epsilon^{1 / 2+\gamma_{1}-\alpha^{\prime}}, \epsilon^{3 / 2}, \epsilon^{\gamma_{1}}, \epsilon^{\alpha+1 / 2}\right) .
\end{aligned}
$$

Extracting the $\epsilon$-independent terms in the limit $\epsilon \rightarrow 0$, we obtain

$$
2 w^{2}\left(t_{1}\right)+1 / 8+4 \int_{0}^{t_{1}} d s_{1}\left(w\left(t_{1}-s_{1}\right)-w\left(t_{1}\right)\right) w^{\prime}\left(s_{1}\right)=0 .
$$

We substitute $w\left(t_{1}\right)=Q\left(t_{1}\right)-\lambda t_{1}$ into this equation. The result becomes (41).

\section{Appendix D. Short time expansion of $Q$}

We assume the form

$$
Q\left(t_{1}\right)=\sum_{k=0}^{\infty} q_{k} t_{1}^{a(2 k-1)}+\lambda t_{1} .
$$

By substituting (D.1) into (41), we can determine $q_{k}(k \geq 1)$ recursively from $q_{0}=c_{0}$. Concretely, the recursion equation becomes

$$
q_{1}=-\frac{1}{64 c_{0}\left(V_{0,1}-1 / 2\right)}
$$

and

$$
q_{k+1}=-\frac{1}{2 q_{0}\left(V_{0, k+1}-1 / 2\right)}\left[\sum_{j=1}^{k} q_{j} q_{k+1-j}\left(V_{j, k+1-j}-1 / 2\right)\right]
$$

for $k \geq 1$, where

$$
V_{m, n}=\frac{p(2 m-1) p(2 n-1)}{p(2 m+2 n-2)}
$$

with $p_{n}=\Gamma(1+a n)$.

\section{References}

[1] Götze W 1984 Z. Phys. B 56139

[2] Leutheusser E 1984 Phys. Rev. A 292765

[3] Crisanti A, Horner H, and Sommers H J 1993 Z. Phys. B 92257

[4] Götze W 1991 Liquids, Freezing and Glass Transition (Les Houches 1989 Session LI) ed J P Hansen 
[5] Fuchs M, Götze W, Hofacker I, and Latz A 1991 J. Phys. Condens. Matter. 35047

[6] Holmes M H 1995 Introduction to perturbation methods (Springer-Verlag, New York)

[7] Bender M and Orszag S A 1999 Advanced Mathematical Methods for Scientists and Engineers, (Springer-Verlag, New York)

[8] Bogoliubov N N and Mitropolsky Y A 1961 Asymptotic Methods in the theory of Nonlinear Oscillations (Goldon and Breach)

[9] Kuramoto Y 1989 Prog. Theor. Phys. Suppl. 99244

[10] Cross M C and Hohenberg P C 1993 Rev. Mod. Phys. 65851

[11] Cornwall J M, Jackiw R, and Tomboulis 1974 Phys. Rev. D 102428

[12] Biroli G and Bouchaud J F, Europhys. Lett. 6721

[13] Iwata M and Sasa S 2007 Europhys. Lett. 7750008

[14] Iwata M and Sasa S 2008 Phys. Rev. E 78 055202(R)

[15] Franz S and Parisi G 2000 J. Phys. Condens. Matter. 126335

[16] Biroli G, Bouchaud J P, Miyazaki K and Reichman D R 2006 Phys. Rev. Lett. 97195701

[17] Culiandolo L F and Kurchan J 1993 Phys. Rev. Lett. 71173

[18] Chamon C and Cugliandolo L F 2007 J. Stat. Mech. P07022

[19] Guckenheimer J and Holmes P 1983 Nonlinear Oscillations, Dynamical Systems and Bifurcations of Vector Fields (Springer-Verlag, New York)

[20] Iwata M and Sasa S 2009 J. Phys. A: Math. Theor. 42075005

[21] Silbert L E, Liu A J, and Nagel S R 2005 Phys. Rev. Lett. 95098301 